\title{
An Exoplanet Transit Observing Method using LCO Telescopes, ExoRequest and astrosource.
}

\author{
Jay Sarva ${ }^{1, *}$, Rachel Freed ${ }^{2}$, Michael Fitzgerald ${ }^{3}$, and Saeed \\ SALIMPOUR ${ }^{4}$
}

${ }^{1}$ Stanford Online High School, California, USA

${ }^{2}$ Institute for Student Astronomical Research, California, USA

${ }^{3}$ Edith Cowan University, Perth, Australia

${ }^{4}$ Deakin University, Melbourne, Australia

"Corresponding author: sarva.jay.s@gmail.com

\begin{abstract}
Exoplanets have become a very active focus of research in the past few years. This is especially true now as the Transiting Exoplanet Survey Satellite (TESS) was launched in April of 2018 and is expected to find thousands of potential candidate exoplanets every year, with the expectation that students and pro-ams will undertake much of the follow-up work. This paper presents a worked example for those intending to get started with observing exoplanets. We analyzed the transit of the planet HAT P56 b utilizing data from Las Cumbres Observatory (LCO) Telescopes and two python scripts. One of the scripts, Exorequest, assists with planning the transit observation while the other, astrosource, automates much of the photometric processing of the transit. While this method uses the LCO data and scheduler, this method will work with any typical optical images.
\end{abstract}

(-) $\circledast \odot 2020$ Astronomy Theory, Observations and Methods Journal

Keywords: Exoplanet astronomy (486), Exoplanet detection methods (489), Astronomical techniques (1684), Astronomical methods (1043)

https://doi.org/10.32374/atom.2020.1.4

\section{INTRODUCTION}

Since the discovery in 1992 of the first exoplanet orbiting a pulsar (Wolszczan \& Frail, 1992) and subsequent 1995 discovery of an exoplanet orbiting a main-sequence star (Mayor \& Queloz, 1995), the study of exoplanets has recently exploded, and it will continue growing exponentially into the future. The growth of this field has led to both the discovery of new exoplanets and detailed analysis of known exoplanets, including study of their atmospheres (Seager
\& Sasselov, 1998) and potential habitability (Seager, 2013).

The radial velocity method and the transit photometry method have been the two detection methods responsible for most of the exoplanet discoveries. The radial velocity method, proposed in 1952 by Otto Struve (Struve, 1952), finds exoplanets through the observation of Doppler shifts in the parent star's spectrum. Transit photometry detects exoplanets through the slight dimming of a star as the planet passes between the star and Earth (Deeg \& Alonso, 2018). 
There have been numerous ground-based searches for exoplanets. For example, WASP (Queloz et al., 2010) is one of the most successful ground-based search programs that uses the transit method, as it has discovered over 100 exoplanets. Another example is HATNet (Bakos, 2002), or the Hungarian Automated Telescope Network, which is a set of six robotic telescopes that has discovered several tens of exoplanets since 2003.

There have also been several space-based searches that have been incredibly successful. The Kepler Mission (Borucki et al., 2010) discovered and confirmed over 2000 exoplanets. After one of its gyroscopes failed, it changed its mission, now referred to as $\mathrm{K} 2$, and discovered more than 2000 exoplanets. It was retired on October 30, 2018 due to fuel depletion. The Transiting Exoplanet Survey Satellite (TESS) (Ricker et al., 2014), launched in April, 2018, also utilizes transit photometry, and is expected to find 20,000 exoplanets within 2 years. All of these exoplanets are catalogued in NASA's Exoplanet Archive, which is freely available to anyone to conduct further analysis. The example in this paper uses data requested from the Las Cumbres Observatory (LCO) global telescope network (Brown et al., 2013). The process can be applied to non-LCO observatories by using Exorequest as a planning tool and undertaking the observations with the different telescope. These images can then be run through the OSS Pipeline (M. Fitzgerald, 2018) to undertake photometry measurements on the images.

This paper intends to present an efficient method for exoplanet transit observation, analysis and modelling, particularly useful for new entrants into the field, for pro-amateurs, as well as those within student research projects (Fitzgerald, Hollow, Rebull, Danaia, \& McKinnon, 2014) and those involved in education programs using the various robotic telescope programs around the world (Gomez \& Fitzgerald, 2017). The process is intended to be as robust as possible providing professional quality results. This paper describes in detail: 1) the selection procedure for picking a target, 2) the short script used to request the images, 3 ) the process used to extract the light curve data from the observed images, and 4) the process used to fit a model transit to the data.

\section{TARGET SELECTION}

As this was the first time the first author had observed an exoplanet transit, an object that would have a high likelihood of successful observing and analysis was picked. The following steps were used to select the target.

1. To select an exoplanet to observe and track, the NASA Exoplanet Archive was used (see Figure $1)$.

2. After clicking on "Confirmed Planets", click on "Select Columns" as seen in Figure 2. Then, check the boxes for Transit Depth, Duration, and Midpoint as seen in Fig 3 and RA and Dec in decimal degrees as seen in Fig 4.

3. An optical magnitude of $<12$ was selected, to ensure the object would be bright enough to image on the 0.4-meter LCO telescopes. These telescopes can easily observe dimmer exoplanets, but longer exposures would be necessary. A rough limit with the present cameras (SBIG STX6303) is 15th magnitude, where the optimal exposure time becomes $\approx 400$ seconds with a w filter.

4. A short period was also queried to allow for multiple attempts to observe the transit. As not every attempt to observe a given transit will be successful, mainly due to weather or competing observers, a shorter period allows more possible attempts within a given time period. This is particularly important as an exoplanet with a longer time between transits might only allow for a small number of periods to be observed within a given timeframe, while a shorter period planet might allow for many more transits to be observed.

5. The search was further narrowed to select for a transit depth of $>1 \%$ as the bigger the transit depth, the more noticable the transit would be on the light curve during analysis. The choice of $1 \%$ is because this is the rough level of scatter for an earth-bound observatory on a typical good night.

6. An appropriate right ascension (RA) of between $[30,90]$ degrees, which contains the optimal RA value for the given time of the year, 60 degrees, was selected. Targets near this value will be in the sky for the longest period of time over the course of the night for any given observatory. We provide a rough list of best RA values for a given time of the year in Table 1. 
There were only a few options of exoplanets that fit all of these criteria to observe, one of which was HAT-P-56 (Hartman et al., 2015). It had a relatively large transit depth, and was very close to the optimal RA. It turns out that HAT-P-56 also has a relatively short transit duration and period, so that meant it was possible to make many observations in a short timespan.

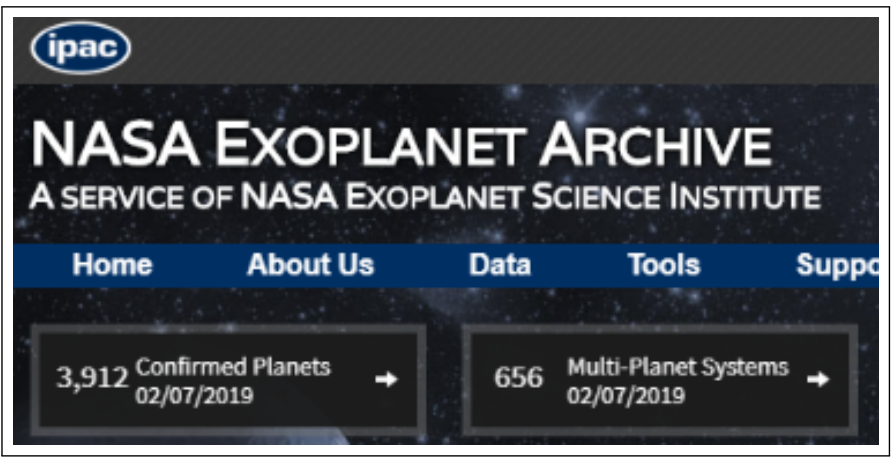

Fig. 1. NASA Exoplanet Archive. Click on "Confirmed Planets."

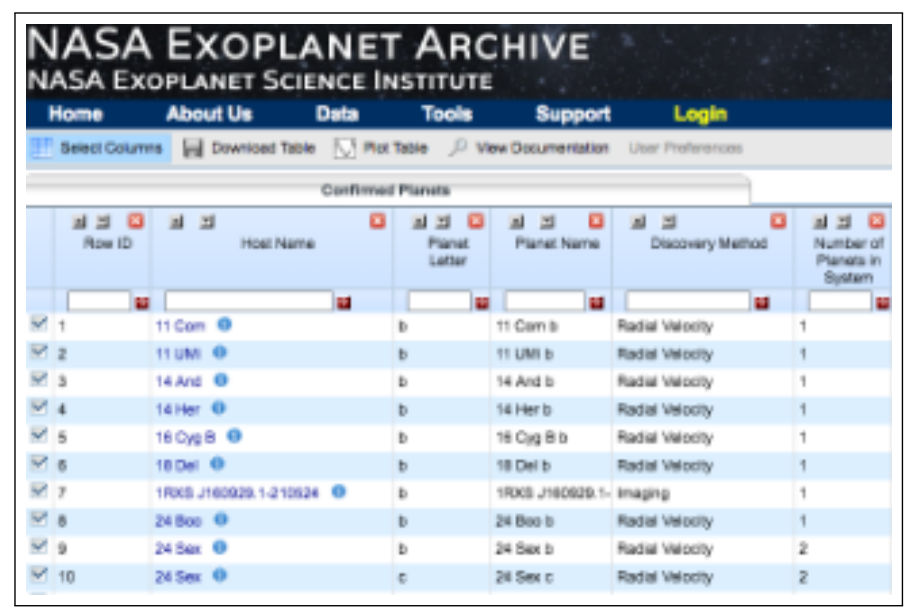

Fig. 2. Click "Select Columns."

\section{USING EXOREQUEST TO REQUEST IMAGES}

Exorequest (available here) is a short python script able to be run online to plan observation times for a given exoplanet transit. It can automatically submit a request to the LCO scheduler, although it can also be used to estimate transit times for any given observatory.

It uses Google Colaboratory for collaborating in Python with other individuals over the internet. To use the version online, a copy will need to be made (File menu -> Save a copy in Drive).

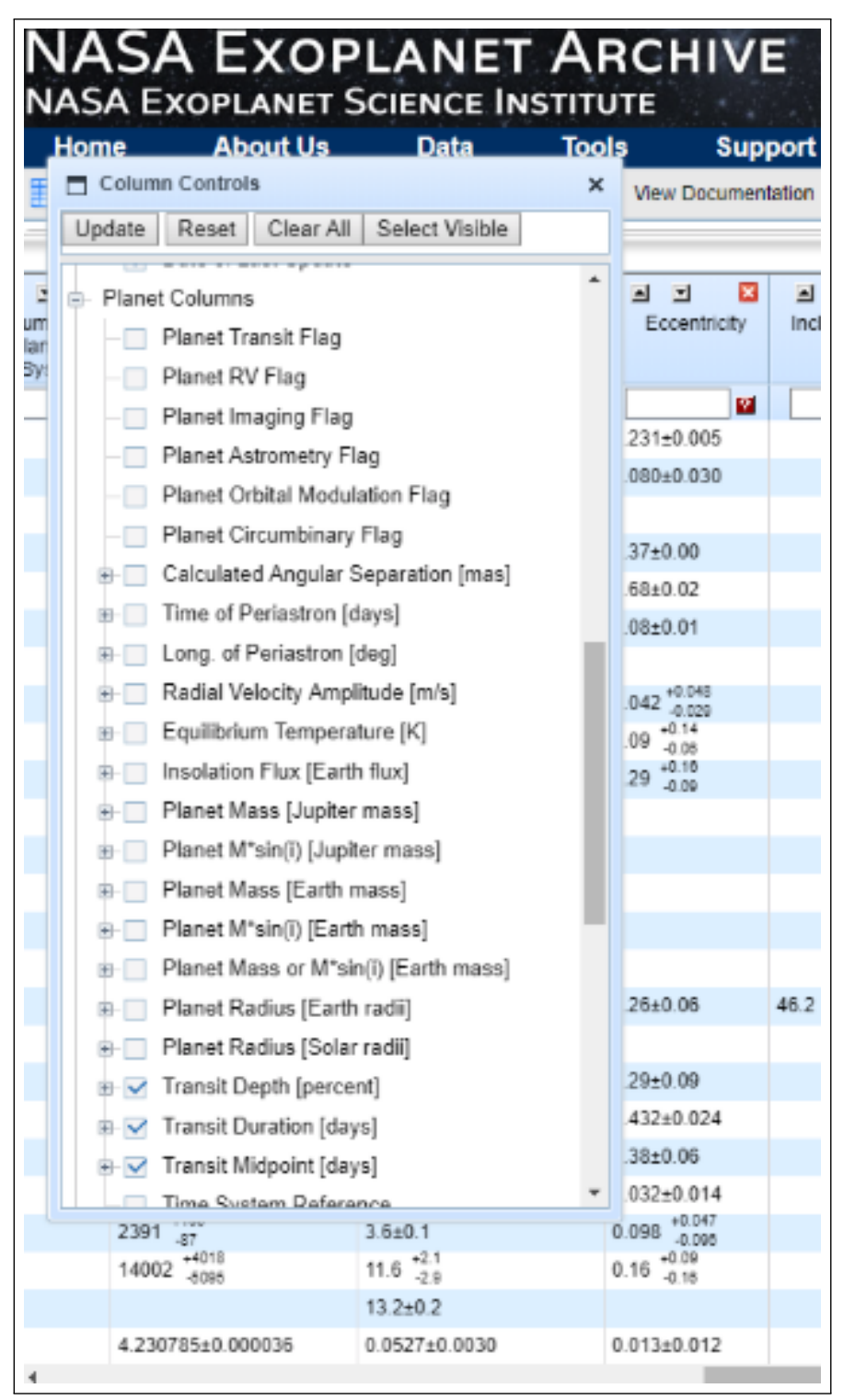

Fig. 3. In the NASA Exoplanet Archive check the boxes for Transit depth, Transit Duration and Transit Midpoint and for RA and Dec (decimal degrees). 
Table 1. Preferable right ascensions based on the time of year in both degrees and hours.

\begin{tabular}{cccccc}
\hline Month & Right Ascension $\left(^{\circ}\right)$ & Month & Right Ascension $\left(^{\circ}\right)$ & Month & Right Ascension $\left({ }^{\circ}\right)$ \\
\hline January & $120(45-195)$ & May & $240(165-315)$ & September & $0(285-75)$ \\
February & $150(75-225)$ & June & $270(195-345)$ & October & $30(315-105)$ \\
March & $180(105-255)$ & July & $300(225-15)$ & November & $60(345-135)$ \\
April & $210(135-285)$ & August & $330(255-45)$ & December & $90(15-165)$ \\
\hline \hline Month & Right Ascension(hrs) & Month & Right Ascension(hrs) & Month & Right Ascension(hrs) \\
\hline January & $8(3-13)$ & May & $16(11-21)$ & September & $0(19-5)$ \\
February & $10(5-15)$ & June & $18(13-23)$ & October & $2(21-7)$ \\
March & $12(7-17)$ & July & $20(15-1)$ & November & $4(23-9)$ \\
April & $14(9-19)$ & August & $22(17-3)$ & December & $6(1-11)$ \\
\hline
\end{tabular}

Table 2. Data needed for ExoRequest to calculate optimal beginning and end times of observation as well as request exoplanet transit images from the Las Cumbres Observatory telescope network.

\begin{tabular}{|c|c|c|}
\hline Object requested & HAT-P-56b & notes \\
\hline \multirow[t]{2}{*}{ Transit Midpoint } & 2456768.50899 & BJD preferably, although \\
\hline & & HJD or JD is approximately correct \\
\hline Transit Duration (hrs) & 2.30 & Use decimal format in hours. \\
\hline Period (days) & 2.790830 & Orbital Period in days \\
\hline \multirow[t]{4}{*}{ Period Multiple } & 1 & This value represents which transit \\
\hline & & in the future you would like to observe. \\
\hline & & 1 is the next transit, 2 is the transit after that, \\
\hline & & 3 is the transit after that and so forth. \\
\hline \multirow[t]{4}{*}{ Optical Mag } & 10.908 & This magnitude is provided in the \\
\hline & & Nasa Exoplanet Archive. \\
\hline & & If $\mathrm{V}$ band is not available \\
\hline & & use the Gaia G band. \\
\hline Exposure Time & 0 & Set to 0 and it will calculate for you. \\
\hline RA & 100.848038 & Use decimal format. \\
\hline Dec & 27.252293 & Use decimal format. \\
\hline
\end{tabular}




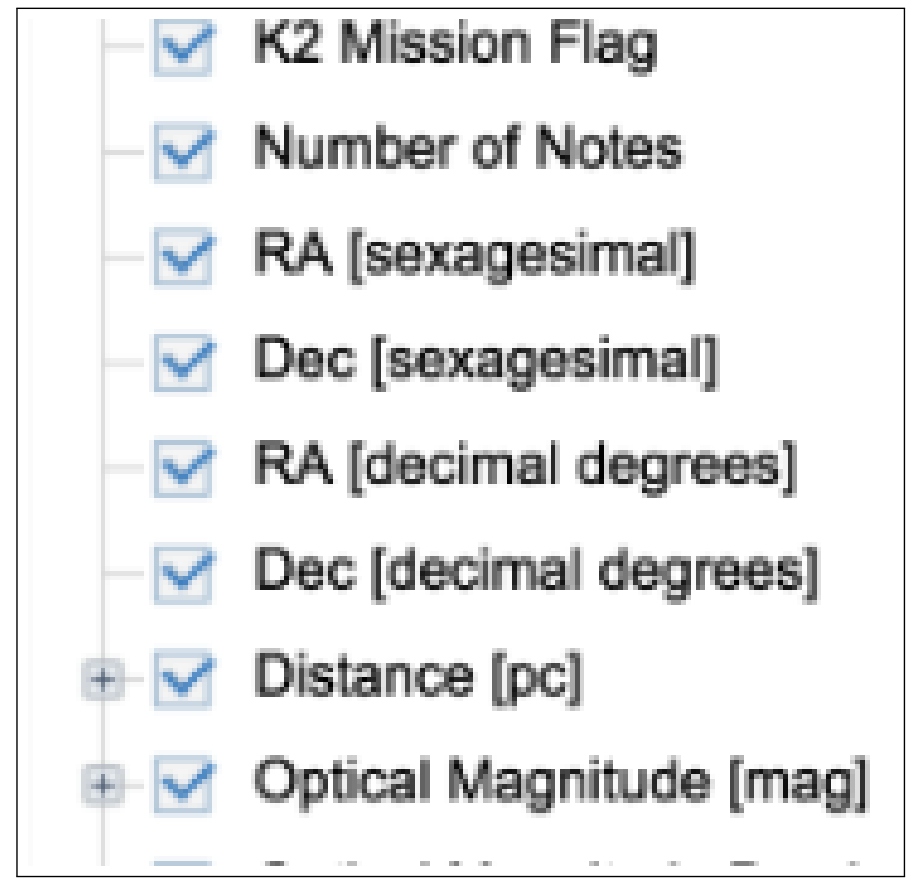

Fig. 4. In the NASA Exoplanet Archive check the boxes for Transit depth, Transit Duration and Transit Midpoint and for RA and Dec (decimal degrees).

Table 2 shows the information that was needed to be input into the ExoRequest script. This information was obtained from the NASA Exoplanet Archive as described above.

Several parameters are necessary to run the ExoRequest script, all of which are noted in Table 2. The transit midpoint, transit duration, period, RA, and Dec can all be found in the various columns of the NASA Exoplanet Archive, which was explored earlier. "Period Multiple" tells the code to pick either the next available transit (1) or the transit after that (2), and so on.

\section{HOW EXOREQUEST WORKS}

Given an estimated period and an estimated time of midtransit, ExoRequest first estimates the centrepoint of the $n^{\text {th }}$ next upcoming transit, where $\mathrm{n}$ is given by the value of "Period Multiple" set by the user. Even with a global telescope network with multiple sites and telescopes, such as LCO, it is usually unlikely that any given transit is situated well in space and time to be observed by any given observatory (or set thereof). For LCO telescopes, the scheduler will tell the user whether it is available or not. For non-LCO telescopes, the user can set the longitude and latitude of the observatory and ExoRequest will indicate if the transit is observable. To find the next available observable transit, the user needs to increase "Period Multiple" by 1 until there is an observable transit.

ExoRequest takes the estimated transit centrepoint $\left(T_{c}\right)$ and uses the provided estimate of the transit duration, requested out-of-eclipse length and the requested buffer to calculate the start and beginning times of the transit observation. A schematic diagram of this is shown in Figure 5. The out-of-eclipse length is the amount of time, set by the user, that should be observed outside of the transit period. This should be an extended period of time as it is necessarily to normalise the transit and to clearly see where the transit starts and ends.

The buffer value is a much smaller value that helps with giving the LCO scheduler a little bit of "wiggle room". For both an LCO and a non-LCO observer, this also makes sure that the transit is still observed even if the timings of the transit center or period were slightly misestimated. ExoRequest will also estimate the exposure time and number of exposures needed if provided a visual magnitude estimate. These values can also be overridden for different filter sets and cameras.

\section{OBSERVATIONS}

Images were obtained by the Las Cumbres Observatory (Brown et al., 2013) 0.4-m telescope located at the McDonald Observatory in Texas, which has been in operation since its installation in April 2012. The network of 0.4-m telescopes were equipped with CCD cameras (SBIG STX6303) with $4 \mathrm{~K} \times 4 \mathrm{~K}$ pixels, each of which is 0.57 arcseconds square. This gives the telescopes a total Field of View (FoV) of $19 \times 29$ arcminutes. In our observations, the w-band filter was used to maximise sensitivity and minimise the exposure time per image. The data came from two separate dates: Dec 7, 2018 (624 images) and Dec. 4, 2018 (426 images).

\section{DATA ANALYSIS}

The data, consisting of 1050 images over two different transit periods were processed by the OSS Pipeline (M. Fitzgerald, 2018). This pipeline automatically reduces the images and performs photometry on each one, providing multiple types of photometry. The type of photometry selected for use in this study was 


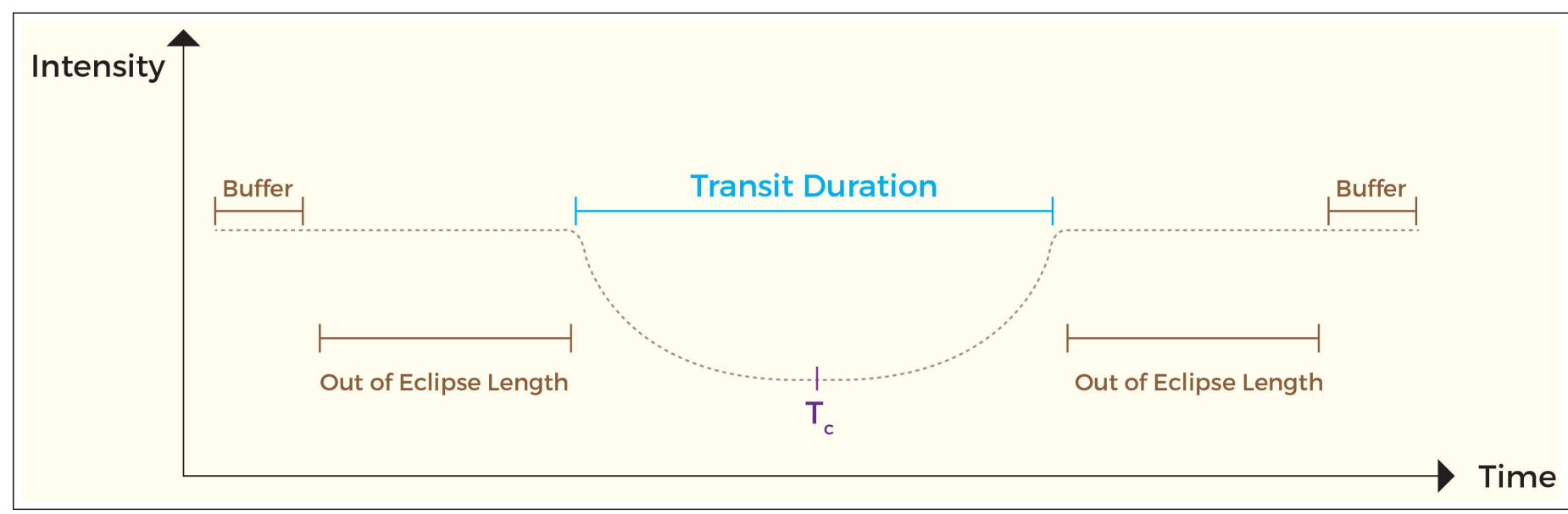

Fig. 5. A schematic diagram of the various aspects that ExoRequest takes into account when choosing a beginning and end time of observation.

PSFEx (Bertin, 2011) Point-Spread-Function (PSF) photometry. PSF photometry, when available and feasible, generally provides a higher signal-to-noise than traditional aperture photometry (Heasley, 1999). The use of the pipeline can be bypassed by using LCO provided images which already contain Source Extractor (Bertin \& Arnouts, 1996) Photometry.

The astrosource python script was used to extract the transit lightcurve from the photometry for each image. The process is described in the following steps:

1. Before starting the process of running each python script, all the observations from a single period must be inserted into the 'inputs' folder.

2. The script sorts through the images and finds the image with the most stars detected by the photometry. That image will be used as a reference frame.

3. After selecting the reference image, the program then sorts through the images and removes the target star as well as any stars that have total counts that are too high (usually 1000000) or too low (usually 10000). This means that stars that have counts that are too high (nearing saturation) and stars with too few counts to achieve a high enough signal-to-noise are both removed.

4. Each image is then reviewed for the number of stars detected. If there are too few stars detected, the image is rejected from the analysis. At the end, the script will output a list of potential comparison stars that have a sufficient brightness and exist in every single image. It will also list out all the images that it plans to use that have not been rejected.

5. The next step picks out the specific comparison stars to be used. Every potential comparison star's variability is measured. Essentially, all the counts of the comparison stars are added up to create a larger comparison star, and every individual comparison star's variability will be compared to the sum of all the comparisons. Then, any star with variability greater than 3 times the standard deviation of the variability is not included in further searches. The process repeats until no comparison stars are rejected and a list of potential comparison stars and their variability is listed.

6. Finally, the potential comparison stars are sorted in order of increasing variability. Comparison stars are then picked from this list in order, until either the total number of counts exceeds $1000000 \mathrm{ADU}$, or if the variability of the star exceeds a specific threshold (in this case, 0.025 mag was used). This leaves us with a list of the least variable, optimal, set of comparison stars to be used with this specific dataset.

7. The second last step essentially generates the light curve for the exoplanet using differential photometry against the sum of the identified comparison stars.

8. The final step is to subtract and even out the baseline of the curve using the out-of-transit observations - removing any slight trends over time 
in the data and normalising the data - as well as remove any outlier data points that might cause some issues when trying to fit a curve. Prior to this detrending, the transit looks like Figure 6. The slight trend can clearly be seen as well as some obviously poor data values at the end of the transit.

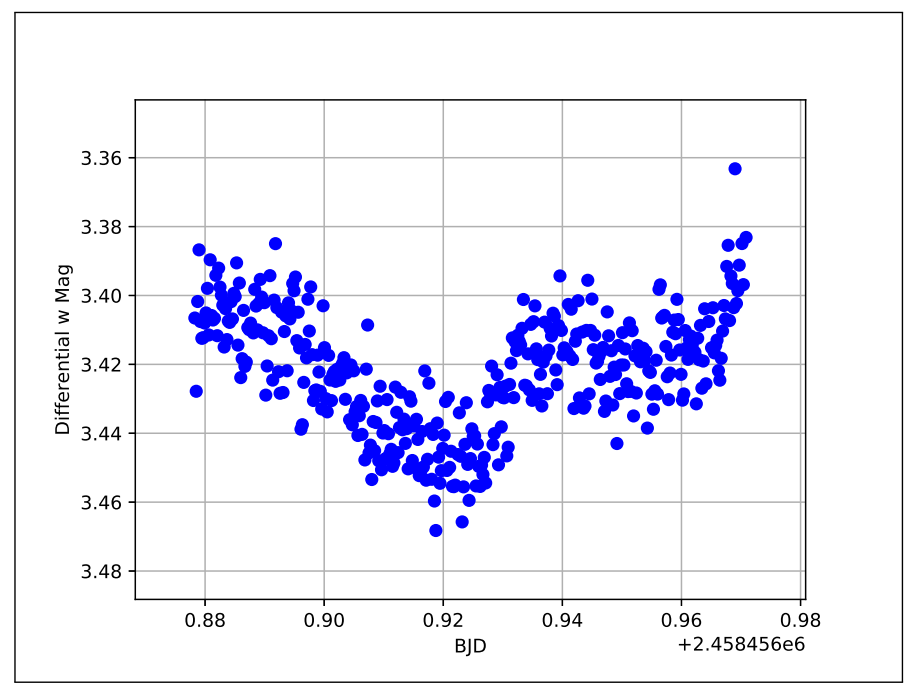

Fig. 6. Light curve for the data from the LCO telescopes from Dec 4, 2018

\section{FITTING A MODEL USING ASTROIMAGEJ}

After producing the transit lightcurve from our data, the next step was to fit a transit model to the light curve. To do this, a version of ImageJ known as AstroImageJ (Collins, Kielkopf, Stassun, \& Hessman, 2017) was utilized. The steps are outlined below.

1. The first step is to ensure that all earlier AIJ preferences for the multiplot function have been reset to default.

2. First, the table with the data from the light curve produced by the astrosource scripts must be opened by clicking "Open Table From File" in the "File" dropdown. Then, in the main window, select X-data to be "Col_1," select Y-data to be "Col_2," check the "Input in Mag" button, and change the colour to Black. Changing the colors will allow for better recognition of the various parts of the fit, and therefore those mentioned in this paper are entirely arbitrary. Finally, ensure to set the "Fit Mode" to the icon that looks like an exoplanet transit.
3. In the Fit Settings window, go to the Plot Settings box. Change the Model line color to "Red" and set the thickness to 3. Furthermore, change both the Residual line color and Symbol Color to purple, and change the shift as much as necessary to clearly separate the two plots such that there is minimal overlap. The settings for the colour and the line thickness are selected to allow for easier analysis of the visualised data.

4. Specify in the same window the estimated period of the exoplanet transit and the radius of the star. It is the radius, not the other values, that is essential as this is used to estimate the true radius of the exoplanet. The other star parameter values have no effect on the measurement.

5. If the orbital eccentricity is known beforehand, uncheck the "Cir" box (which would automatically assume a circular orbit) and enter in the eccentricity (and if available, the omega value).

6. Next, uncheck the "Auto Update Priors" box. Go to the EXOFAST - Quadratic Limb Darkening website which interpolates the (Claret \& Bloemen, 2011) quadratic limb darkening tables and enter the temperature value, the $\log (\mathrm{g})$ value and the $[\mathrm{Fe} / \mathrm{H}]$ value for your star as well as selecting the filter used. Clicking submit will output two values. The left value is for Quad LD u1 and the right value is for Quad LD u2 which can then be entered into the fit window. If sufficient information about the star is not available, select both Quad LD u1 and Quad LD u2 as 0.3, which is the default value. At this point, Quad LD u1 and Quad LD u2 can be set to Lock.

7. Set Baseline Flux (Raw) to lock and uncheck use. Change the value of Prior Centre until the outof-transit lightcurve data roughly lines up with 1.00 on the y-axis.

8. Set $T_{c}$ to Lock and uncheck use. Change the value of prior center until it is roughly the value of the center of the transit and unlock it.

9. At this point, it will be necessary to adjust Prior Centers for $\mathrm{Rp} / \mathrm{R}^{*}, \mathrm{a} / \mathrm{R}^{*}, \mathrm{Tc}$ and Inclination until a suitable model is autofit. The key is to attempt to minimize the residual RMS value. You may need to lock or unlock some of these values at various times. 
10. Once a reasonable fit has been acquired, in the "Fit Settings" window, go to file menu and select "save fit results as text file", this will save the results of your curve to a text file. The most important parameter is the "Planet Radius (Rjup)" which provides the radius of the planet in terms of the radius of Jupiter.

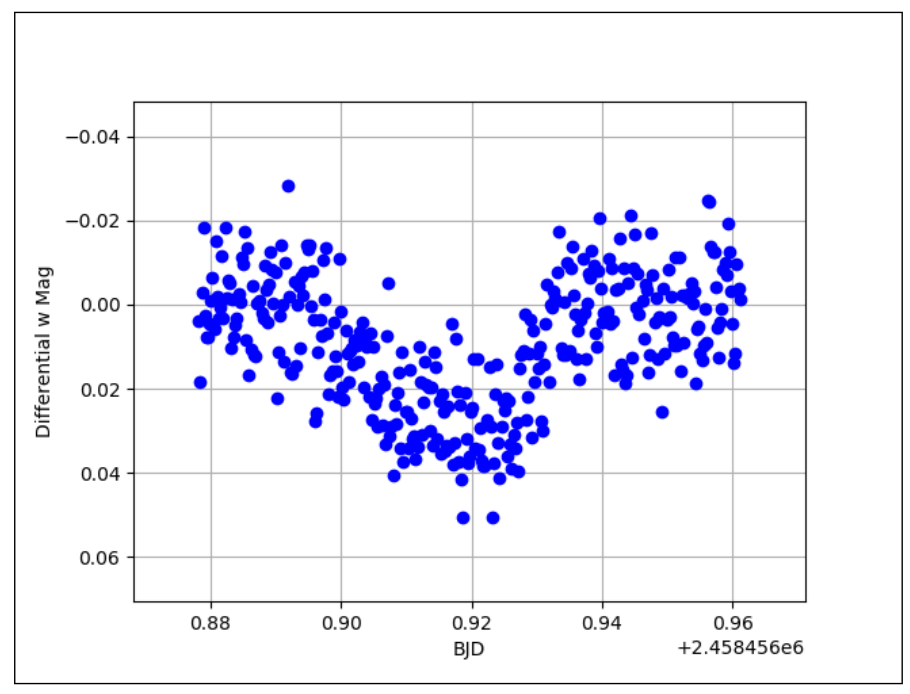

Fig. 7. Light curve for the data from the LCO telescopes from Dec 4, 2018

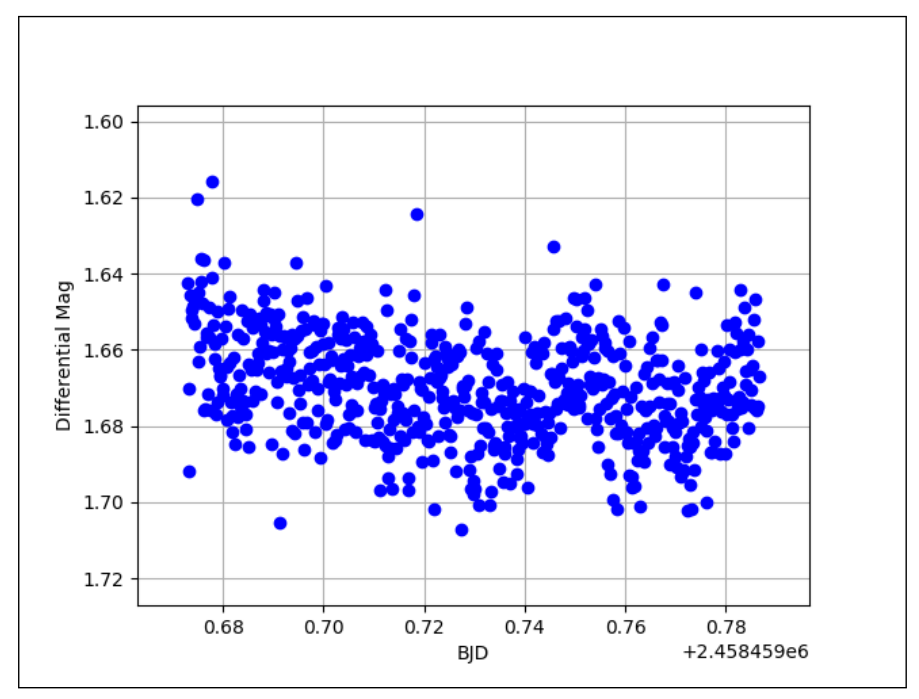

Fig. 8. Light curve for the data from the LCO telescopes from Dec 7, 2018. The data from this period appeared to be significantly more noisy and less easily decipherable compared to the other transit.

\section{RESULTS}

We took observations of two different transits. The first lightcurve observed is shown in Figure 7, and

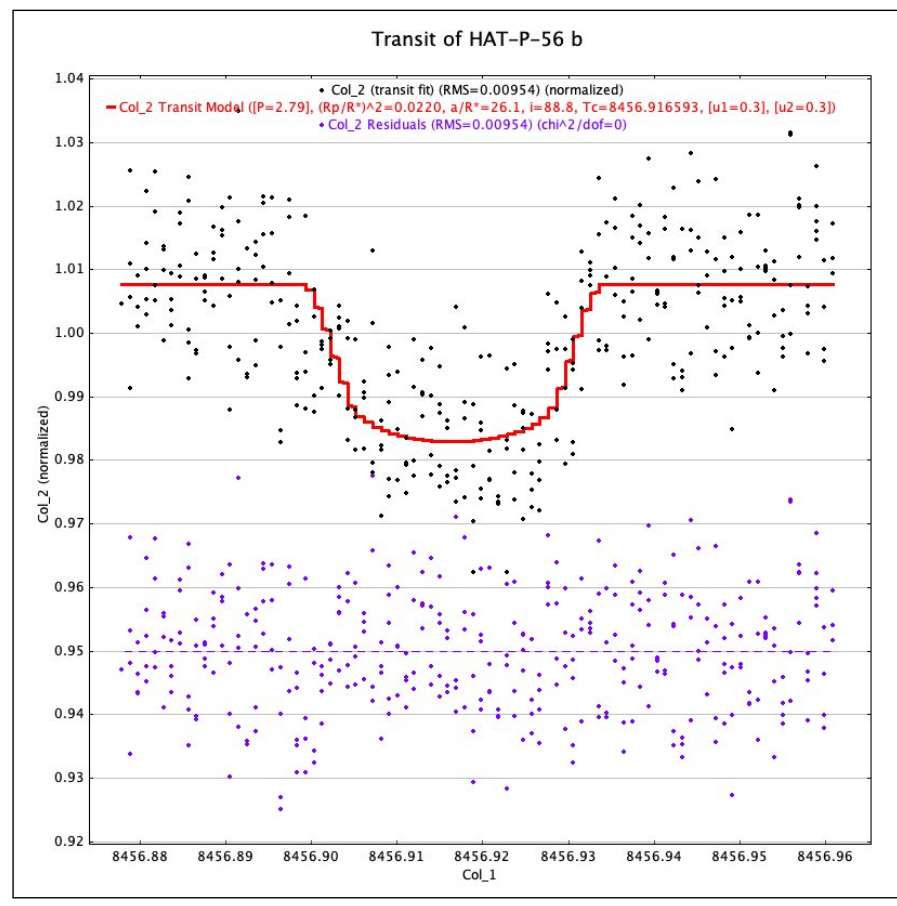

Fig. 9. Fit reached through AstroImageJ.

the second lightcurve observed is shown in Figure 8. The first light curve shows a distinct dip, which is representative of a transiting object. The second curve was not the expected shape of a transit, as it had a slight unexpected bump in the middle of the dip and a much larger scatter in the data.

The lower quality data in the December 7th data was likely due to poor weather and seeing at the site at that time. This illustrates the point that even when observations are made at high quality locations with professional instrumentation, the high quality requirements when observing exoplanet transits may be thwarted by conditions outside of the observer's control, such as the weather. When attempting to fit the second lightcurve with a transit model in AstroImageJ, a reasonable fit was not obtained due to the quality of the data.

The December 4th transit lightcurve and the resulting fit are shown in Figure 9. It has a relatively even baseline alongside a very "clean" dip. The planet radius calculated based on the fit settings we used here was $1.45 R_{j}$, which is quite close to the published radius of HAT-P-56b of $1.466 R_{j}$ (Huang et al., 2015).

\section{DISCUSSION}

It is particularly difficult to undertake exoplanet transit observations for beginning astronomers, and this paper provides a simplified approach. This method 
enables pro-ams, students and teachers to pursue amateur exoplanet research with a robust approach that can eventually contribute to TESS follow-up and other exoplanet projects, such as the Exoplanet Timing Survey (Zellem et al., 2020).

In particular, the use of the OSS Pipeline (M. Fitzgerald, 2018) removes the necessity of undertaking manual flat/bias/dark reduction (although images provided by LCO are already corrected for these instrumental signatures). The OSS Pipeline automatically fixes header or WCS issues (and automatically estimates a WCS where missing) as well as calculates a variety of new useful header items. A particularly useful example of header adjustment for exoplanets is that the BJD is calculated and inserted directly into the header and into a human-readable filename. The use of the pipeline saves many hours of tedious processing. It also performs all of the photometry necessary, providing 3 PSF types and 3 aperture-based photometry methods, all undertaken with professional level software.

The use of ExoRequest allows the accurate planning (and requesting, if using LCO) of a given exoplanet transit. For a beginner, there is a lot to think about when the transit occurs, when to begin and end observing as well as whether the transit can be clearly observed at a given site, date and time. For a more advanced user, ExoRequest still acts to streamline the selection and submission process for a given transit.

Through the use of astrosource, many of the timeconsuming considerations with regards to differential photometry are taken care of as well as removing multiple sources of potential mistakes and bias. For instance, astrosource selects the least variable set of stars within the provided data (as well as rejecting known variable stars), allowing a selection of the absolute best comparison stars in any field within a specific set of data. This makes the selection of comparison stars rapid and also the most optimised, which is simply not possible with manual selection of comparison stars. Astrosource also provides a detrend function that removes any slight trends apparent in an exoplanet lightcurve using the out-of-transit data.

The photometric output of astrosource can be imported directly into AstroImageJ to use the inbuilt modelling functionality that is available there. There are plans in future to explore some of the more advanced transit modelling packages available in python. However, AstroImageJ is well known in the pro-am community for exoplanet analysis and is a good graphical way to explore modelling the transit lightcurve.

\section{CONCLUSION}

Overall, through the use of ExoRequest, the astrosource scripts and AstroImageJ, we were able to produce a robust plot of a transit lightcurve of the exoplanet HAT-P-56 b. We did have to reject one of the two observed transits due to poor seeing and weather conditions at the time of observation. This method streamlines many of the time-consuming processes for planning, observing and analysing a given exoplanet transit, provides professional quality results while removing multiple layers of human-induced error and bias.

\section{ACKNOWLEDGMENTS}

This work makes use of observations from the LCO global telescope network. Telescope time was provided by the Global Sky Partners program at LCO.

This research has made use of the NASA Exoplanet Archive, which is operated by the California Institute of Technology, under contract with the National Aeronautics and Space Administration under the Exoplanet Exploration Program.

\section{REFERENCES}

Bakos, G. A. (2002). "system description and first light curves of the hungarian automated telescope, an autonomous observatory for variability search". , 114(799), 974-987.

Bertin, E. (2011, July). Automated Morphometry with SExtractor and PSFEx. In I. N. Evans, A. Accomazzi, D. J. Mink, \& A. H. Rots (Eds.), Astronomical data analysis software and systems $x x$ (Vol. 442, p. 435).

Bertin, E., \& Arnouts, S. (1996). Sextractor: Software for source extraction. Astronomy and Astrophysics Supplement Series, 117(2), 393-404.

Borucki, W. J., Koch, D., Basri, G., Batalha, N., Brown, T., Caldwell, D., ... others (2010). Kepler planet-detection mission: introduction and first results. Science, 327(5968), 977-980.

Brown, T., Baliber, N., Bianco, F., Bowman, M., Burleson, B., Conway, P., ... others (2013). Las cumbres observatory global telescope network. Publications of the Astronomical Society of the Pacific, 125(931), 1031. 
Claret, A., \& Bloemen, S. (2011, May). Gravity and limb-darkening coefficients for the Kepler, CoRoT, Spitzer, uvby, UBVRIJHK, and Sloan photometric systems. , 529, A75. doi:

Collins, K. A., Kielkopf, J. F., Stassun, K. G., \& Hessman, F. V. (2017, Feb). AstroImageJ: Image Processing and Photometric Extraction for Ultraprecise Astronomical Light Curves. , 153(2), 77. doi:

Deeg, H. J., \& Alonso, R. (2018). Transit photometry as an exoplanet discovery method. Handbook of exoplanets, 633-657.

Fitzgerald, Hollow, R., Rebull, L. M., Danaia, L., \& McKinnon, D. H. (2014). A review of high school level astronomy student research projects over the last two decades. Publications of the Astronomical Society of Australia, 31.

Fitzgerald, M. (2018). The our solar siblings pipeline. RTSRE Proceedings, 1(1), 347-358.

Gomez, E. L., \& Fitzgerald, M. T. (2017). Robotic telescopes in education. Astronomical Review, 13(1), 28-68.

Hartman, J. D., Bhatti, W., Bakos, G. Á., Bieryla, A., Kovács, G., Latham, D. W., ... others (2015). Hat-p-50b, hat-p-51b, hat-p-52b, and hat-p-53b: three transiting hot jupiters and a transiting hot saturn from the hatnet survey. The Astronomical Journal, 150(6), 168.

Heasley, J. N. (1999, Jan). Point-Spread Function Fitting Photometry. In E. R. Craine, D. L. Crawford, \& R. A. Tucker (Eds.), Precision ccd photometry (Vol. 189, p. 56).

Huang, C. X., Hartman, J. D., Bakos, G. Á., Penev, K., Bhatti, W., Bieryla, A., ... Sári, P. (2015, Sep). HAT-P-56b: An Inflated Massive Hot Jupiter Transiting a Bright F Star Followed Up with K2 Campaign 0 Observations. , 150(3), 85. doi:

Mayor, M., \& Queloz, D. (1995). A jupiter-mass companion to a solar-type star. Nature, 378(6555), 355-359.

Queloz, D., Anderson, D. R., Collier Cameron, A., Gillon, M., Hebb, L., Hellier, C., ... West, R. (2010, July). WASP-8b: a retrograde transiting planet in a multiple system. , 517, L1. doi:

Ricker, G. R., Winn, J. N., Vanderspek, R., Latham, D. W., Bakos, G. Á., Bean, J. L., ... others (2014). Transiting exoplanet survey satellite. Journal of Astronomical Telescopes, Instruments, and Systems, 1(1), 014003.

Seager, S. (2013). "exoplanet habitability". Science,
340(6132), 577-581. doi:

Seager, S., \& Sasselov, D. D. (1998). Extrasolar giant planets under strong stellar irradiation. The Astrophysical Journal Letters, 502(2), L157.

Struve, O. (1952, October). Proposal for a project of high-precision stellar radial velocity work. The Observatory, 72, 199-200.

Wolszczan, A., \& Frail, D. A. (1992). A planetary system around the millisecond pulsar psr1257 + 12. Nature, 355(6356), 145-47.

Zellem, R. T., Pearson, K. A., Blaser, E., Fowler, M., Ciardi, D. R., Biferno, A., ... others (2020). Utilizing small telescopes operated by citizen scientists for transiting exoplanet follow-up. Publications of the Astronomical Society of the Pacific, 132(1011), 054401. 